\title{
PDK4 Deficiency Induces Intrinsic Apoptosis in Response to Starvation in Fibroblasts from Doberman Pinschers with Dilated Cardiomyopathy
}

\author{
Kathryn Taggart, ${ }^{1,2}$ Amara Estrada, ${ }^{1, *}$ Patrick Thompson, ${ }^{1}$ Francisco Lourenco, ${ }^{1,2}$ Sara Kirmani, ${ }^{2}$ \\ Silveli Suzuki-Hatano, ${ }^{2}$ and Christina A. Pacak ${ }^{2}$
}

\begin{abstract}
The Doberman pinscher (DP) canine breed displays a high incidence of idiopathic, nonischemic dilated cardiomyopathy (DCM) with increased mortality. A common mutation in DPs is a splice site deletion in the pyruvate dehydrogenase kinase 4 (PDK4) gene that shows a positive correlation with DCM development. PDK4, a vital mitochondrial protein, controls the switch between glycolysis and oxidative phosphorylation based upon nutrient availability. It is likely, although unproven, that DPs with the PDK4 mutation are unable to switch to oxidative phosphorylation during periods of low nutrient availability, and thus are highly susceptible to mitochondrial-mediated apoptosis. This study investigated cell viability, mitochondrial stress, and activation of the intrinsic (mitochondrial mediated) apoptotic pathway in dermal fibroblasts from DPs that were healthy (PDK4 ${ }^{\mathrm{wt} / \mathrm{wt}}$ ), heterozygous $\left(\right.$ PDK4 $\left.{ }^{\text {wt/del }}\right)$, and homozygous (PDK4 ${ }^{\text {del/del) }}$ ) for the PDK4 mutation under conditions of high (unstarved) and low (starved) nutrient availability in vitro. As hypothesized, PDK4 ${ }^{\text {wt/del }}$ and PDK4 ${ }^{\text {del/del }}$ cells showed evidence of mitochondrial stress and activation of the intrinsic apoptotic pathway following starvation, while the PDK4 ${ }^{\text {wt/wt }}$ cells remained healthy and viable under these conditions. Adeno-associated virus (AAV) PDK4-mediated gene replacement experiments confirmed cause-effect relationships between PDK4 deficiency and apoptosis activation. The restoration of function observed following administration of AAV-PDK4 provides strong support for the translation of this gene therapy approach into the clinical realm for PDK4-affected Dobermans.
\end{abstract}

Keywords: AAV; cardiomyopathy; dilated Doberman Pinschers; mitochondria; PDK4; pyruvate dehydrogenase kinase

\section{Introduction}

The Doberman Pinscher (DP) canine breed displays a high incidence of idiopathic, nonischemic, dilated cardiomyopathy (DCM) with elevated mortality. Pedigree evaluations of affected dogs suggest the disease is inherited as an autosomal dominant trait. DCM in the DP breed typically progresses until the dog dies of heart failure or sudden death due to arrhythmias. ${ }^{1-5}$ The DP model is an excellent model to study human nonischemic DCM due to the similarity in growth, size, and disease progression. ${ }^{6}$
Thus far, there are two published studies suggesting specific genetic loci that are associated with the DCM phenotype in DPs. ${ }^{7-9}$ One is a 16 base pair deletion in the $5^{\prime}$ donor splice site of intron 10 of the pyruvate dehydrogenase kinase 4 (PDK4) gene. Although this trait displays incomplete penetrance and this particular mutation does not account for the entire population of DPs that develop DCM, the correlation is significant, and regular cardiac evaluations in dogs known to carry the mutation in one or both alleles are recommended. ${ }^{10,11}$ PDK4 is a mitochondrial protein that

${ }^{1}$ Department of Small Animal Clinical Sciences, College of Veterinary Medicine, University of Florida, Gainesville, Florida.

${ }^{2}$ Department of Pediatrics, College of Medicine, University of Florida, Gainesville, Florida.

*Address correspondence to: Amara Estrada, DVM, DACVIM, Department of Small Animal Clinical Sciences, College of Veterinary Medicine, University of Florida, PO Box 100126, Gainesville, FL 32608, E-mail: estradaa@ufl.edu

(C) Kathryn Taggart et al. 2017; Published by Mary Ann Liebert, Inc. This is an Open Access article distributed under the terms of the Creative Commons Attribution License, which permits unrestricted use, distribution, and reproduction in any medium, provided the original work is properly cited. 
plays an important role in metabolic flexibility by enabling mitochondria to adjust primary fuel source usage based on nutrient availability.

Although there are four different PDK isoforms, PDK4 is the predominant form expressed in cardiac tissue. In a postprandial state, the pyruvate dehydrogenase complex (PDC) converts pyruvate, the product of glycolysis, to acetyl-CoA to be utilized by the citric acid cycle. When blood glucose levels are low, the PDC becomes phosphorylated by PDK4 and the cell is required to use oxidative phosphorylation instead of glycolysis for energy production. ${ }^{12}$ As cardiomyocytes must produce synchronous, energy-demanding ventricular contractions, oxidative phosphorylation is the preferred method of energy generation because of the higher levels of ATP it yields. ${ }^{13}$

DPs deficient in PDK4, however, are unable to switch off the PDC and thus continue to use glycolysis. Over time, the constant reliance upon glycolysis for energy production becomes detrimental, as these hearts are unable to generate sufficient energy for long-term healthy cardiac function. ${ }^{14}$ The switch away from fuel sources, preferred by the mature heart (fatty acids), and toward glucose, which (along with lactate) is preferred by the immature heart, is a common feature among all cardiomyopathies and believed to exacerbate cardiac remodeling that ultimately manifests as ventricular enlargement, arrhythmias, and ineffective contractions. ${ }^{15}$

For this study, skin biopsies were obtained to isolate dermal fibroblasts from DPs that were prescreened for DCM with echocardiograms, Holter monitoring, and a genetic test for the PDK4 splice site mutation. We have previously shown that fibroblasts from DPs with phenotypic features of DCM that were heterozygous $\left(\mathrm{PDK} 4^{\mathrm{wt} / \mathrm{del}}\right)$ or homozygous $\left(\mathrm{PDK} 4^{\mathrm{del} / \mathrm{del}}\right)$ for the PDK4 mutation displayed significantly reduced oxygen consumption rates compared to healthy control cells from DPs without phenotypic features of DCM and negative for the PDK4 mutation (PDK4 $\left.{ }^{\mathrm{wt} / \mathrm{wt}}\right) .{ }^{16}$ Our preliminary experiments suggested that glucose deprivation led to decreased viability in cells deficient for PDK4. The purpose of this study was to determine the apoptotic pathway responsible for this cell death.

As PDK4 suppression of the PDC complex is crucial for conservation of glucose and facilitation of fatty acid oxidation when glucose is scarce, we hypothesized that $\mathrm{PDK} 4^{\mathrm{del} / \mathrm{del}}$ cells display a markedly decreased viability when deprived of glucose due to induction of the intrinsic (mitochondrially mediated) apoptotic pathway. As reactive oxygen species (ROS) levels and caspase-9 activity are typically increased during oxidative stress and activation of the intrinsic apoptotic pathway, we expected that the homozygous mutant (PDK4 $4^{\mathrm{del} / \mathrm{del}}$ ) cells would show the highest levels of these indicators and subsequently, the highest rates of apoptosis when cultured under glucose-free conditions. ${ }^{17,18}$

In the heterozygous cells (PDK4 $4^{\mathrm{wt} / \mathrm{del}}$ ), we expected more moderate levels of ROS and caspase- 9 and a lower rate of apoptosis compared to the homozygous cells, as we expect they retain some ability to phosphorylate the PDC. Finally, we expected our healthy control $\left(\mathrm{PDK} 4^{\mathrm{wt} / \mathrm{wt}}\right)$ cells would remain viable under glucosefree conditions, show little to no apoptosis, and generate only low levels of ROS. To test our hypotheses, we evaluated cell viability, markers of mitochondrial stress, and caspase-9 activity and expression. The cause-effect relationship between the PDK4 deficiency and apoptosis was further confirmed through adeno-associated virus (AAV) mediated PDK4 gene replacement experiments.

\section{Materials and Methods}

University of Florida veterinarians performed examinations on DPs that included echocardiograms, 24-h Holter monitoring, and a genetic test for the PDK4 splice site mutation. All procedures met and maintain the highest ethical and welfare standards in all areas involving interactions with animals. Dermal biopsies were obtained according to IACUC standards and primary fibroblasts were isolated as previously described. ${ }^{19}$ Fibroblasts from dogs representing $\mathrm{PDK} 4^{\mathrm{del} / \mathrm{wt}}, \mathrm{PDK} 4^{\mathrm{del} / \mathrm{del}}$, and $\mathrm{PDK}^{\mathrm{wt} / \mathrm{wt}}$ genotypes were plated as necessary for each experiment. For maintenance, cells were grown at $37^{\circ} \mathrm{C}$ in $5 \% \mathrm{CO}_{2}$ in DMEM (Corning 10-013CV) $+20 \%$ fetal bovine serum (FBS) and $1 \%$ Pen/Strep.

\section{Viability assays}

Cells were counted before being plated at 50,000 cells per well in a 24 -well plate with 8 wells per genotype. After $4 \mathrm{~h}$, the cells had adhered and the media were changed. Half of the cells were maintained in a maintenance medium (unstarved) and the other half in FBS and a glucose-free medium (starved). After $24 \mathrm{~h}$, the cells were detached with trypsin, centrifuged, and suspended in fresh medium, and viable cells quantified with an automatic cell counter.

\section{AAV administration}

Fibroblasts were seeded and half the wells from each line were treated with AAV-CBA-PDK4 and the other half were labeled with a green fluorescent 
protein (GFP) for visualization, AAV-CBA-GFP, at a multiplicity of infection of 50,000 viral genomes per cell. They were incubated for 7 days with medium changes every other day. Cells were then detached and plated into 96-well plates with 10,000 cells/well. Four hours after seeding, the medium was changed so that half the cells from each condition were starved and the other half remained unstarved. Each experiment was performed after $24 \mathrm{~h}$ of starvation.

TaqMan gene expression assays

Total RNA was isolated using the Quick-RNA Mini-prep kit (Zymo Research) according to the manufacturer's protocol. The gDNA was removed using DNase I (Zymo Research). Quantitative real-time-PCR was performed on an Applied Biosystems Step-One Plus Real-Time PCR System using TaqMan primer/probes designed to detect PDK4 (Cf02648648_m1; Thermo Scientific) and RPS19BP1 (Cf02640781_g1; Thermo Scientific).

ROS assay

After starvation, the medium was aspirated and for each condition, half the wells were treated with $20 \mu \mathrm{M}$ of rotenone (a positive control for ROS production) and incubated for $1 \mathrm{~h}$. The medium was aspirated and all wells rinsed with $1 \times$ phosphate-buffered saline. Five micromolars of dihydroethidium (DHE) was added and cells were incubated for $20 \mathrm{~min}$ before microplate analysis.

\section{Mitochondrial toxicity assay}

After starvation, the medium was aspirated and the Mitochondrial ToxGlo Assay (Cat. No. G800; Promega) was performed as indicated in the kit protocol to evaluate cytotoxicity (fluorescence) and ATP production (luminescence). A sample of each cell line was treated with Digitonin $(80 \mu \mathrm{g} / \mathrm{mL})$ as a positive control for cytoxicity. ${ }^{20}$

\section{Caspase- 9 assay}

After starvation, the medium was aspirated and the Caspase-Glo 9 Assay (Cat. No. G8211; Promega) was performed as indicated in the kit protocol. Following a 45-min incubation period, luminescence was determined using a microplate reader.

Protein determination

Following each assay, total protein was quantified in each well and all experimental results were normalized to protein.

\section{Immunofluorescence}

Representative fibroblasts from each condition were seeded onto cover-slips and fixed using $4 \%$ paraformaldehyde. They were stained with TOMM20 anti-Rabbit (HPA011562, 1:100; Sigma) and Cytochrome C antiMouse (ab110528, 1:100; Abcam) or Capase-9 anti-rabbit (NB10056119, 1:100; Novus Bio) and Anti-Alexa Fluor 488 phalloidin (A12379, 1:50; Life Technologies). They were then incubated in corresponding secondary antibodies (AlexaFluor rabbit 488 A11034, rabbit 568 A11036, mouse 488 A11029, and mouse 568 A11031 [Life Technologies] 1:500).

\section{Data processing}

Data analyses, graph creation, and statistical analyses were performed using Sigma Plot Software. There were four biological replicates for each condition and experiment, and each biological sample was tested in triplicate. Descriptive statistics and paired $t$-tests were performed on all data.

\section{Results}

Our first experiment was designed to compare viability between PDK $4^{\mathrm{wt} / \mathrm{wt}}, \mathrm{PDK} 4^{\mathrm{wt} / \mathrm{del}}$, and PDK $4^{\mathrm{del} / \mathrm{del}}$ fibroblast lines in unstarved and starved medium conditions. Phase-contrast images of fibroblasts from each line in the maintenance medium (unstarved) showed healthy monolayers of cells. In contrast, after $24 \mathrm{~h}$ of starvation, $\mathrm{PDK} 4^{\mathrm{wt} / \mathrm{wt}}$ fibroblasts displayed slightly altered morphology, PDK $4^{\text {wt/del }}$ fibroblasts appeared more altered and fewer in number, and PDK4 ${ }^{\mathrm{del} / \mathrm{del}}$ fibroblasts were rounded and far less healthy in appearance (Fig. 1A-C).

All fibroblasts displayed high viability with no statistically significant difference in cell numbers following $24 \mathrm{~h}$ of growth in the maintenance medium (Fig. 1D). As expected, the starved PDK4 ${ }^{\text {wt/del }}$ and PDK4 ${ }^{\mathrm{del} / \mathrm{del}}$ fibroblast lines displayed a significant decrease in viable cell numbers compared to the starved PDK $4^{\mathrm{wt} / \mathrm{wt}}$ fibroblasts. The PDK4 ${ }^{\mathrm{del} / \mathrm{del}}$ fibroblasts showed the most severe response to starvation as there was a statistically significant decrease in starved cell numbers compared to the same line in the maintenance medium. These results show that a deficiency in PDK4 function results in altered morphology and decreased viability in response to nutrient deprivation.

To confirm that the PDK4 splice site mutation was the cause for the decreased viability in response to starvation, we used AAV to deliver a healthy copy of canine PDK4 driven by a strong, ubiquitous chick beta 

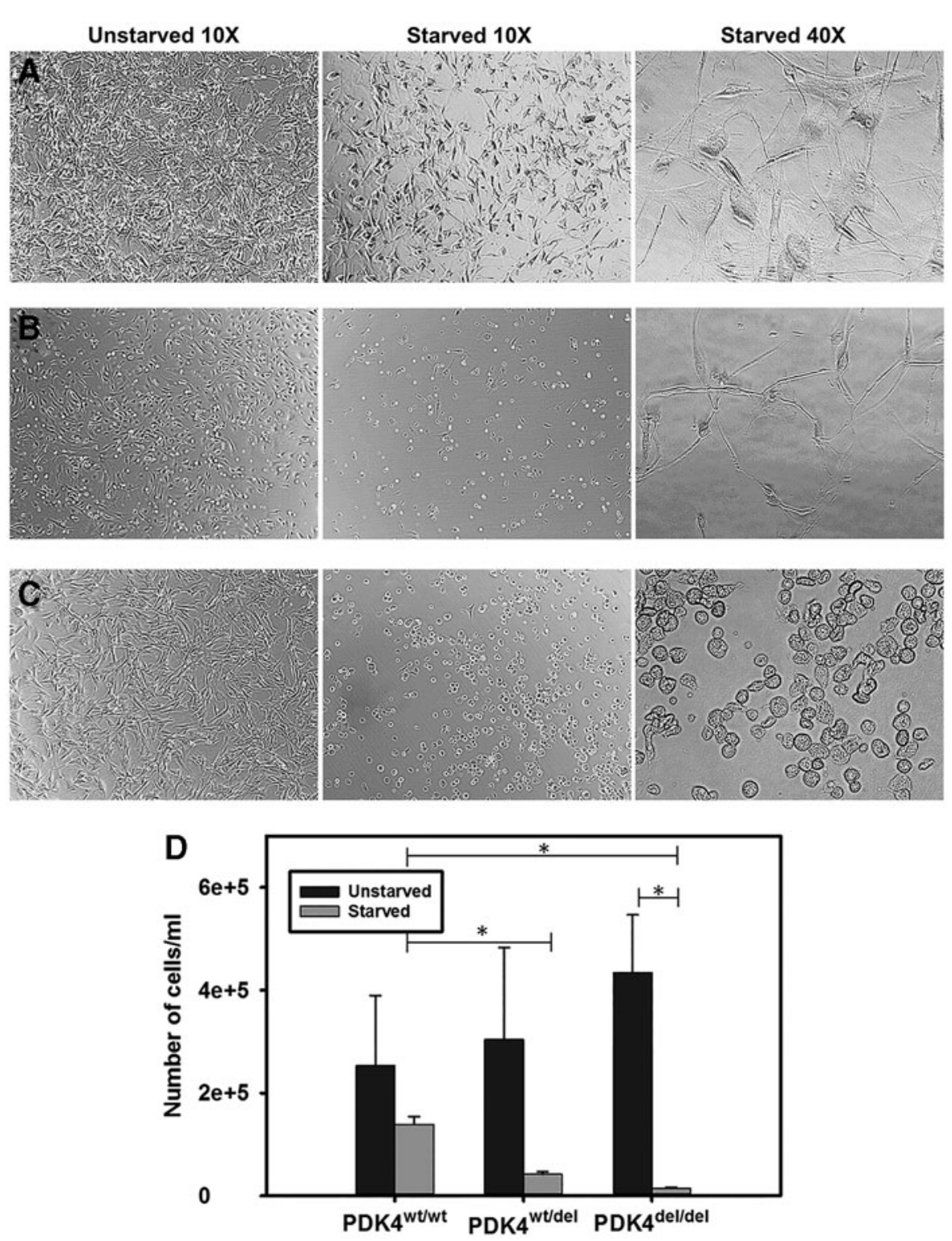

FIG. 1. Reduced cell viability in PDK4-deficient cells. Phase contrast light microscopy images of (A) PDK4 ${ }^{\text {wt/wt }}$, (B) PDK4 ${ }^{\mathrm{wt} / \mathrm{del}}$, and (C) PDK4 ${ }^{\mathrm{del} / \mathrm{del}}$ fibroblasts incubated in unstarved and starved conditions. (D) A significant decrease in cell numbers was observed in starved PDK4-deficient fibroblasts compared to healthy controls $\left(\mathrm{PDK} 4{ }^{\mathrm{wt} / \mathrm{del}} p=0.03\right)\left(\mathrm{PDK} 4{ }^{\mathrm{del} / \mathrm{del}} p=0.015\right)$. PDK4, pyruvate dehydrogenase kinase $4 .{ }^{*} p \leq 0.05$.

actin (CBA) promoter to PDK4 ${ }^{\mathrm{wt} / \mathrm{del}}$ and PDK4 $4^{\mathrm{del} / \mathrm{del}}$ fibroblasts using a previously described quadruple mutant of the AAV2 capsid, which has been determined to successfully transduce primary cells in vitro (AAVCBA-PDK4). ${ }^{20}$ Healthy PDK ${ }^{\mathrm{wt} / \text { wt }}$ fibroblasts and untreated PDK4 ${ }^{\text {wt/del }}$ and PDK4 ${ }^{\text {del } / \text { del }}$ control fibroblasts were all transduced with an identical vector expressing the GFP marker transgene in place of PDK4 (AAV-
CBA-GFP) (Fig. 2A, B). TaqMan gene expression assays to compare PDK4 RNA transcript levels showed a 300400 fold increase in PDK4 expression in AAV-PDK4treated fibroblasts compared to untreated (AAV-GFP) controls (Fig. 2C).

Quantification of starved PDK4 $4^{\mathrm{wt} / \mathrm{del}}$ and PDK4 ${ }^{\mathrm{del} / \mathrm{del}}$ fibroblasts revealed an increase in cell numbers in AAV-PDK4-treated cells compared to those that were 
administered the AAV-GFP control vector (Fig. 2D). Thus, our AAV-PDK4 vector successfully transduced the fibroblasts, generated PDK4 RNA transcripts, and improved cell viability in response to starvation.

Several experiments were performed to compare unstarved (AAV-GFP), starved (AAV-GFP), and starved (AAV-PDK4) treated fibroblasts represent-

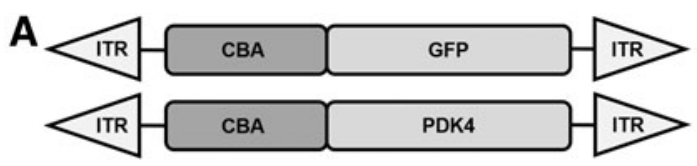

B Day 1: Seeded cells

Day 2: AAV treatment (MOI 50,000)

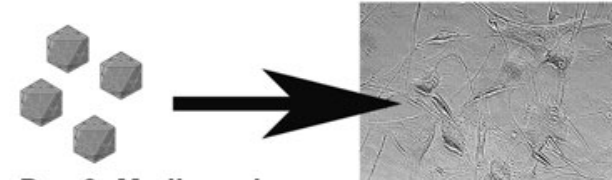

Day 3: Medium change

Day 5: Medium change

Day 7: Counted and replated cells for assays, after 4 hrs changed medium to + or glucose

Day 8: Performed experiments
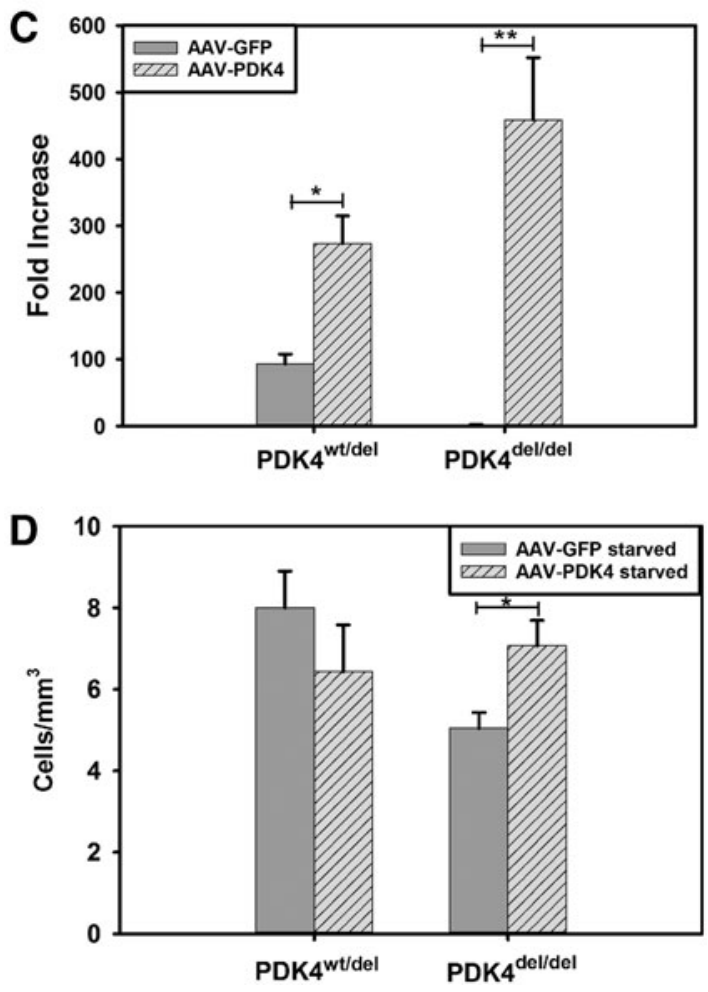

ing each genotype in a variety of assays to better understand the specific mechanism of cell death in response to nutrient deprivation. A MitoTox assay revealed a significant increase in mitochondrial toxicity in starved samples compared to the same lines in the normal (unstarved) maintenance medium. The most dramatic increase in toxicity was observed in the starved PDK $4^{\mathrm{del} / \mathrm{del}}$ cells with levels that were significantly higher than starved $\mathrm{PDK}^{\mathrm{wt} / \mathrm{wt}}$ cells $(p<0.001)$. There was no difference between the AAV-GFP-treated PDK $4^{\mathrm{wt} / \mathrm{wt}}$ or PDK $4^{\mathrm{wt} / \mathrm{del}}$ cells in starved or unstarved conditions. The starved AAVPDK4-treated PDK4 ${ }^{\text {del/del }}$ fibroblasts showed a statistically significant decrease in toxicity compared to those same cells treated with the AAV-GFP control virus $(p=0.004)$ (Fig. 3A).

As an increased ATP production is associated with cell death during apoptosis, intracellular ATP was determined in each fibroblast line and condition. ${ }^{21}$ Increased levels of ATP were found in the starved $\mathrm{PDK} 4^{\mathrm{wt} / \mathrm{del}}$ and $\mathrm{PDK} 4^{\mathrm{del} / \mathrm{del}}$ cells compared to unstarved controls and PDK $4^{\mathrm{wt} / \mathrm{wt}}$ fibroblasts $(p<$ $0.001)$. The administration of AAV-PDK4 to starved $\mathrm{PDK} 4^{\mathrm{wt} / \mathrm{del}}$ and $\mathrm{PDK} 4^{\mathrm{del} / \mathrm{del}}$ cells successfully decreased intracellular ATP levels $(p<0.001)$ (Fig. 3B).

ROS molecules were quantified using dihydroethidium (DHE), a superoxide indicator that exhibits blue fluorescence in the cytosol until oxidized, where it intercalates within the DNA and fluoresces red in the nucleus. In microplate assays, the PDK $4^{\mathrm{wt} / \mathrm{del}}$ and PDK4 ${ }^{\text {del/del }}$ fibroblast lines displayed significantly higher levels of ROS following starvation compared to unstarved

FIG. 2. AAV-mediated Gene Delivery. (A) A diagram of the vector constructs used in this study-control vector GFP driven by a CBA promoter (top-AAV-GFP) and therapeutic vector canine PDK4 driven by a CBA promoter (bottom-AAV-PDK4). Both promoter-transgene constructs are situated between AAV ITR to enable viral capsid packaging. (B) Timeline protocol of AAV treatment and fibroblast starvation. (C) Graph showing high level expression of PDK4 RNA transcripts in AAV-PDK4-treated PDK $4^{\text {wt/del }}$ and PDK4 $4^{\text {del/del }}$ fibroblasts compared to controls ( ${ }^{*} p<0.05$ and ${ }^{* *} p<0.01$ respectively). (D) Graph showing increased PDK4 ${ }^{\mathrm{del} / \mathrm{del}}$ fibroblast viability in starvation conditions following AAV-PDK4 treatment $\left({ }^{*} p<0.05\right)$. AAV, adeno-associated virus; $C B A$, chick beta actin; GFP, green fluorescent protein; ITR, inverted terminal repeats. 

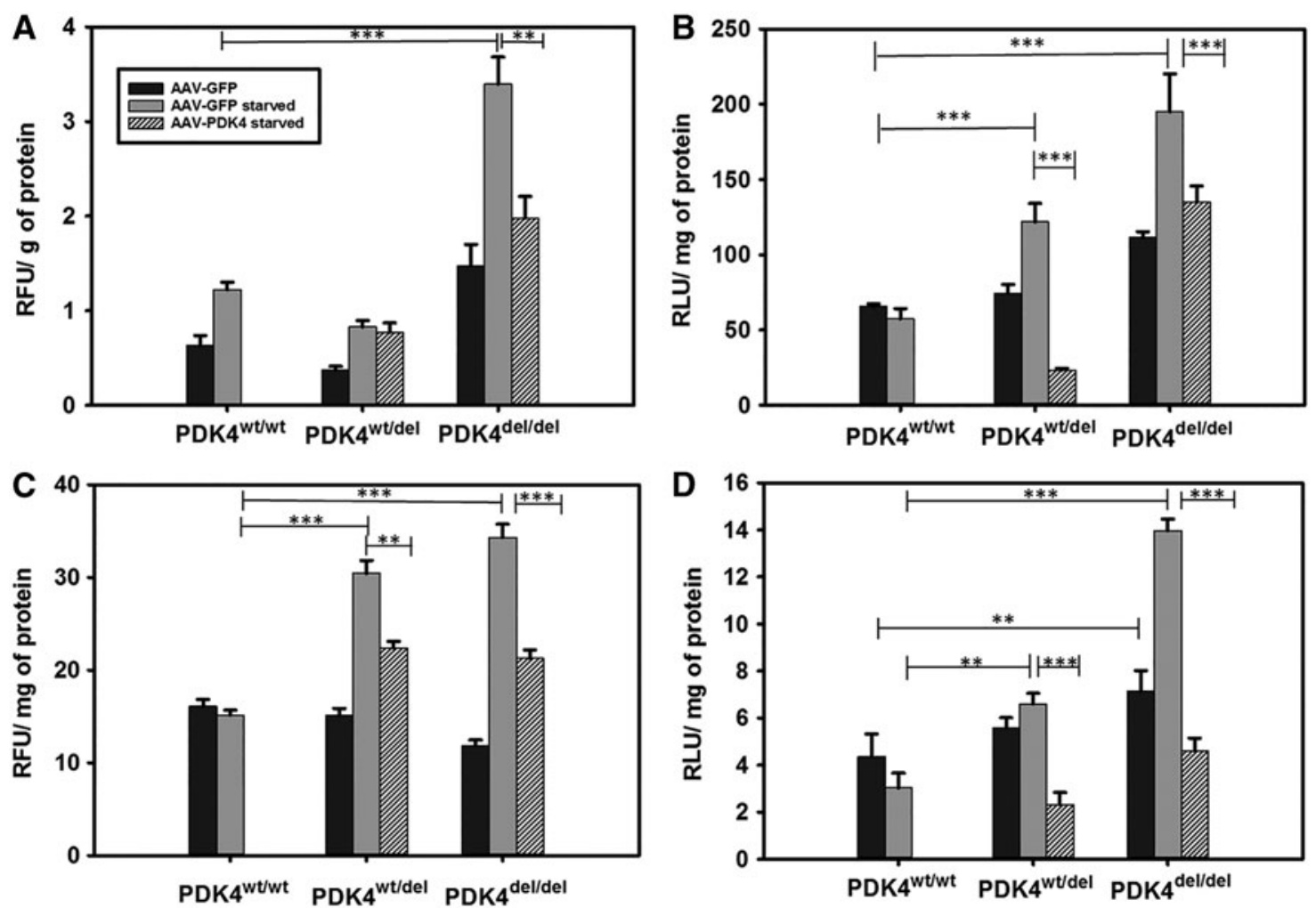

FIG. 3. Increased mitochondrial stress and activation of intrinsic apoptotic signaling in PDK4 deficient cells. (A) Increased mitochondrial toxicity was observed in starved PDK4 ${ }^{\text {del/del }}$ fibroblasts $\left({ }^{* *} p<0.01\right)$. The toxicity level decreased to unstarved levels following administration of AAV-PDK4 $\left({ }^{* *} p<0.001\right)$. (B) An increase in ATP production was observed in starved PDK4 ${ }^{\mathrm{wt} / \mathrm{del}}$ and PDK4 ${ }^{\mathrm{del} / \mathrm{del}}$ fibroblasts. ATP levels decreased following administration of AAV-PDK4 ( $\left.{ }^{* *} p<0.001\right)$. (C) An increase in ROS production was observed in starved PDK4 ${ }^{\text {wt/del }}$ and PDK4 ${ }^{\text {del } / d e l}$ fibroblasts $\left({ }^{* *} p<0.01\right)$. ROS levels decreased following administration of AAV-PDK4 ( $\left.^{* * *} p<0.001\right)$. (D) An increase in caspase-9 activity levels was observed in starved PDK4 ${ }^{\mathrm{wt} / \mathrm{del}}$ and PDK4 ${ }^{\mathrm{del} / \mathrm{del}}$ fibroblasts (** $p<0.01$ ). Caspase- 9 levels decreased following the administration of AAV-PDK4 (*** $p<0.001)$. ROS, reactive oxygen species.

conditions, whereas the PDK $4^{\mathrm{wt} / \mathrm{wt}}$ fibroblasts showed no difference in ROS levels. The starved PDK $4^{\text {wt } / \mathrm{del}}$ and PDK $4^{\text {del/del }}$ fibroblasts responded well to AAV-PDK4 administration, which dramatically reduced their ROS levels compared to AAV-GFP controls $(p<0.01$ and $p<0.001$, respectively).

To determine whether or not the intrinsic (mitochondrial mediated) apoptotic pathway is activated through starvation of the PDK4-deficient cells, a microplate-based caspase- 9 quantification assay was performed. As expected, the starved $\mathrm{PDK} 4^{\mathrm{wt} / \mathrm{del}}$ and $\mathrm{PDK}^{\mathrm{del} / \mathrm{del}}$ fibroblasts displayed significantly increased levels of caspase- 9 compared to the PDK $4^{\mathrm{wt} / \mathrm{wt}}$ cells $(p=0.008$ and $p<0.001$ respectively). By far, the highest caspase-9 levels were observed in starved PDK4 ${ }^{\mathrm{del} / \mathrm{del}} \mathrm{fi}-$ broblasts, indicating that these homozygous cells are strongly activated to undergo mitochondrial-mediated apoptosis when starved (Fig. 3D). When fibroblasts were treated with AAV-PDK4 and then starved for $24 \mathrm{~h}$, we observed a significant decrease in caspase- 9 levels in both the $\mathrm{PDK} 4^{\mathrm{wt} / \mathrm{del}}$ and $\mathrm{PDK}^{\mathrm{del} / \mathrm{del}}$ cells $(p<0.001)$. Importantly, AAV-PDK4-treated and AAV-PDK4-starved PDK4 ${ }^{\mathrm{wt} / \mathrm{del}}$ and PDK $4^{\mathrm{del} / \mathrm{del}}$ fibroblasts showed no statistical difference in caspase- 9 levels compared to starved PDK $4^{\mathrm{wt} / \mathrm{wt}}$ cells. Taken together, these results imply that, while mitochondrial stress is clearly activated in PDK4-deficient cells during periods of starvation, this response is dramatically 


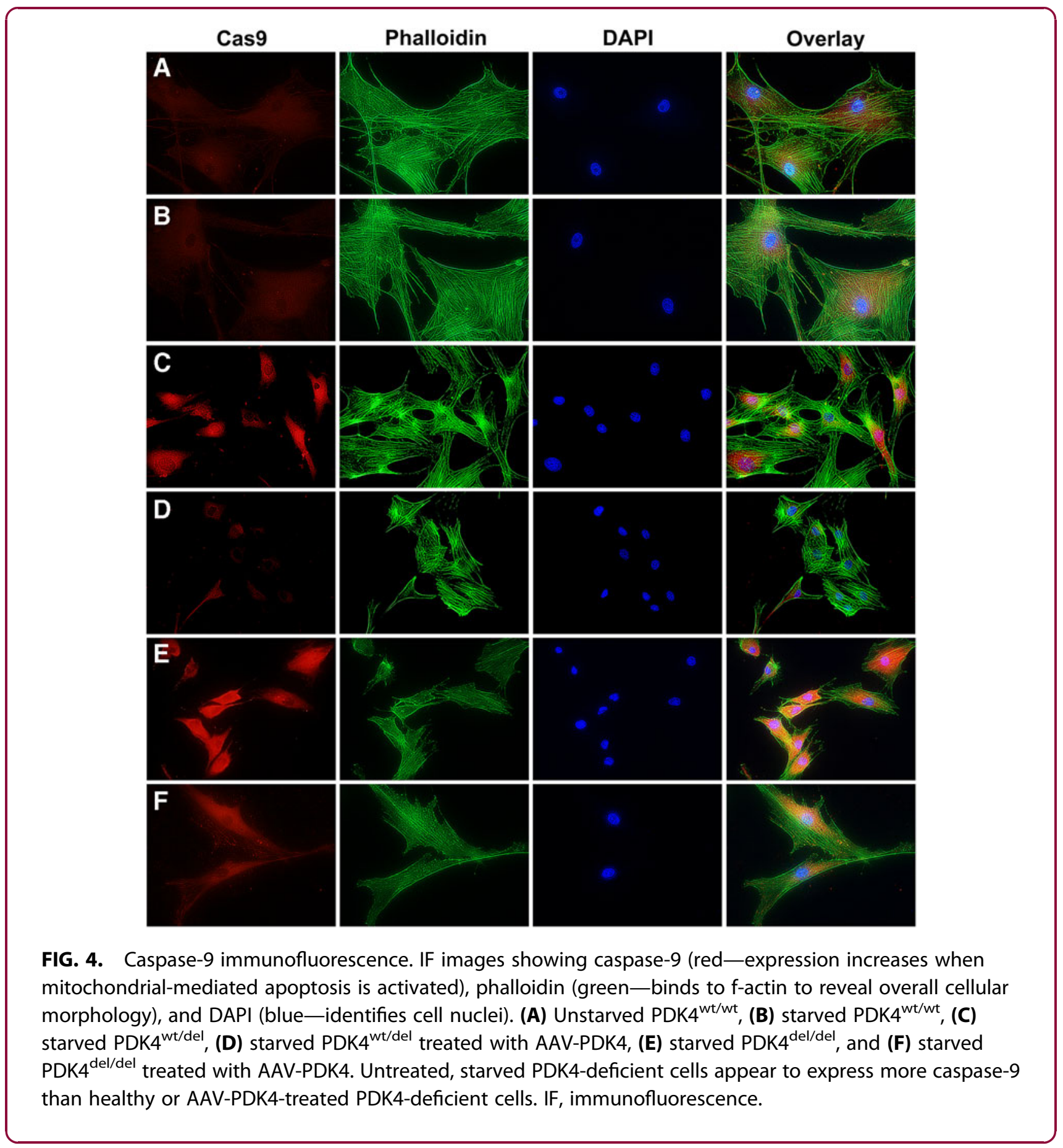

blunted in those same lines, following the administration of healthy copies of PDK4.

Immunofluorescence (IF) staining for caspase-9 in starved fibroblasts that were either untreated or administered AAV-PDK 4 mimicked the caspase- 9 assay results by showing an increased caspase- 9 expression in untreated starved $\mathrm{PDK} 4^{\mathrm{wt} / \mathrm{del}}$ and $\mathrm{PDK}^{\mathrm{del} / \mathrm{del}}$ cells compared to those that were treated, which appeared similar to healthy PDK4 ${ }^{\mathrm{wt} / \mathrm{wt}}$ cells (Fig. 4). IF staining for TOMM20 was performed to reveal mitochondrial distribution within cells. Our results showed no discernable difference between unstarved and starved healthy $\mathrm{PDK} 4^{\mathrm{wt} / \mathrm{wt}}$ cells (Fig. 5A, B), while mitochondria in untreated starved $\mathrm{PDK}^{\mathrm{wt} / \mathrm{del}}$ and $\mathrm{PDK}^{\mathrm{del} / \mathrm{del}}$ cells showed a tendency toward 


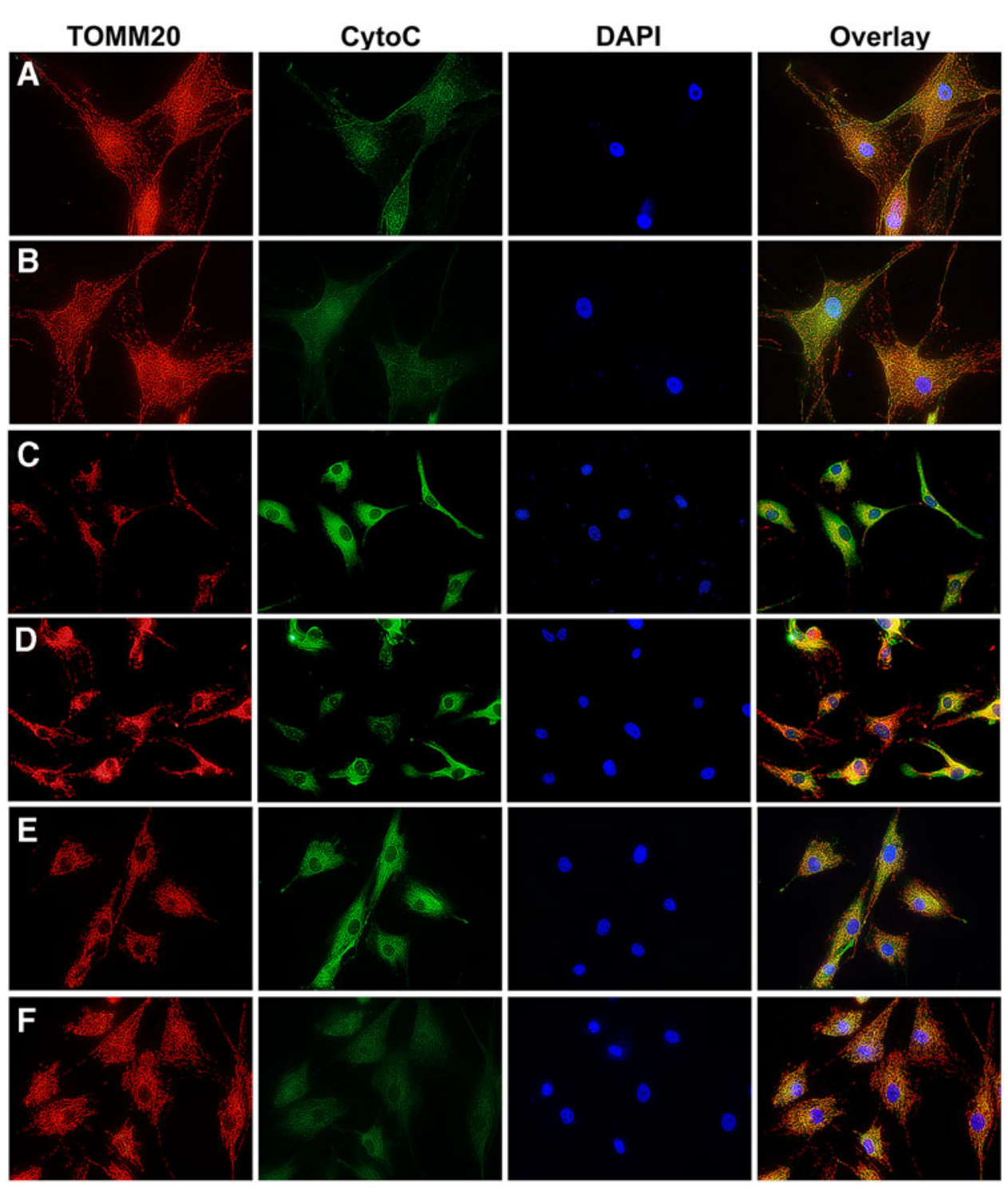

FIG. 5. TOMM 20 and cytochrome $C$ immunofluorescence. IF images showing TOMM20 (red-identifies mitochondrial distribution within cells), CytoC (green-expression increases and is released from mitochondrial when apoptosis is activated), and DAPI (blue-identifies cell nuclei). (A) Unstarved PDK4 ${ }^{\text {wt } / w t}$, (B) starved PDK4 ${ }^{\mathrm{wt} / \mathrm{wt}}$, (C) starved PDK4 ${ }^{\mathrm{wt} / \mathrm{del}}$, (D) starved PDK4 ${ }^{\mathrm{wt} / \mathrm{del}}$ treated with AAV-PDK4, (E) starved PDK4 ${ }^{\mathrm{del} / \mathrm{del}}$, and (F) starved PDK4 ${ }^{\text {del/del }}$ treated with AAV-PDK4. Untreated, starved PDK4-deficient cells appear to express more cytochrome $\mathrm{C}$ and tend to display a more perinuclear distribution of mitochondria than healthy or AAV-PDK4-treated PDK4-deficient cells.

perinuclear localization. This appeared to normalize in AAV-PDK4-treated starved PDK4 $4^{\text {wt/del }}$ and $\mathrm{PDK}^{\mathrm{del} / \mathrm{del}}$ fibroblasts (Fig. 4C-F). Finally, as the release of cytochrome $\mathrm{C}$ from mitochondria is a major activator of caspase-dependent apoptotic signaling, IF staining for cytochrome $\mathrm{C}$ was performed and revealed an in- creased expression in starved untreated $\mathrm{PDK} 4^{\mathrm{wt} / \mathrm{del}}$ and $\mathrm{PDK}^{\mathrm{del} / \mathrm{del}}$ fibroblasts, which were also reduced in AAV-PDK4 treated cells (Fig. 4C-F). In sum, these results provide further evidence that the cell death observed in PDK4-deficient cells is a result of the intrinsic mitochondrial-mediated apoptotic pathway. 


\section{Discussion}

Our results firmly demonstrate that deficient PDK4 function is consequential during periods of low nutrient availability. While PDK4 $4^{\mathrm{wt} / \mathrm{wt}}$ fibroblasts are able to adjust to periods of stress through activation of PDK4 and subsequent phosphorylation of the PDC, PDK4 ${ }^{\mathrm{wt} / \mathrm{del}}$ and PDK4 ${ }^{\mathrm{del} / \mathrm{del}}$ fibroblasts display difficulty in surviving starvation. As PDK4 is a crucial mitochondrial kinase, we hypothesized that the cell death observed in PDK $4^{\text {wt } / \mathrm{del}}$ and $\mathrm{PDK} 4^{\mathrm{del} / \mathrm{del}}$ cells was triggered by the intrinsic mitochondrial-mediated apoptotic pathway.

The increases in mitochondrial toxicity, intracellular ATP generation, and ROS levels in PDK4-deficient fibroblasts compared to healthy PDK $4^{\mathrm{wt} / \mathrm{wt}}$ cells in response to starvation indicate that the mitochondria of these cells are at the very least, negatively impacted by their inability to switch from glycolysis to oxidative phosphorylation. Dramatic increases in caspase-9 activity and caspase-9 IF staining in starved PDK4 ${ }^{\mathrm{wt} / \mathrm{del}}$ and PDK $4{ }^{\mathrm{del} / \mathrm{del}}$ fibroblasts, in addition to corresponding increases in cytochrome-c staining compared to healthy PDK $4^{\mathrm{wt} / \mathrm{wt}}$ cells, further confirmed activation of the intrinsic apoptotic pathway.

The results from our PDK4 gene replacement studies showed improved cellular viability and reduced mitochondrial toxicity, ROS generation, and caspase-9 activation in response to starvation in AAV-PDK4-treated fibroblasts that were deficient for PDK4. In addition to confirming the causeeffect relationship between deficient PDK4 function and mitochondrial-mediated apoptosis, these data also provide strong support for the translation of this gene therapy approach into PDK $4^{\mathrm{del} / \mathrm{del}} \mathrm{DPs}$ in the clinic.

In sum, we have demonstrated the importance of PDK4 function during periods of low nutrient availability. Our observed activation of the intrinsic apoptotic pathway in fibroblasts deficient for PDK4 suggests that cardiomyocytes in the hearts of $\mathrm{PDK} 4^{\mathrm{wt} / \mathrm{del}}$ and PDK $4^{\mathrm{del} / \mathrm{del}}$ DPs face a similar metabolic challenge. While these dogs rarely experience true nutrient deprivation, the inability to effectively switch from glycolysis to oxidative phosphorylation likely has minor initial consequences that evolve over time into cardiac remodeling and ultimately result in DCM and heart failure. Further investigations into the specific diets of affected and unaffected DPs with identical mutation statuses may reveal a relation- ship between the development of DCM and nutrition in this at-risk population.

\section{Acknowledgments}

The authors thank The Doberman Pinscher Club of America, Cooper's Fund, University of Florida Vector Core, and Dr. Arun Srivastava for kindly sharing the AAV2 quad-mutant capsid; and the Boehringer Ingelheim Veterinary Scholars Fund supported this study.

\section{Author Disclosure Statement}

No competing financial interests exist.

\section{References}

1. Wess G, Schulze A, Butz V, et al. Prevalence of dilated cardiomyopathy in Doberman Pinschers in various age groups. J Vet Intern Med. 2010;24: 533-538.

2. O'Grady MR, O'Sullivan ML. Dilated cardiomyopathy: an update. Vet Clin North Am Small Anim Pract. 2004;34:1187-1207.

3. Calvert CA, Jacobs GJ, Smith DD, et al. Association between results of ambulatory electrocardiography and development of cardiomyopathy during long-term follow-up of Doberman Pinschers. J Am Vet Med Assoc. 2000;216:34-39.

4. Calvert CA, Jacobs G, Pickus CW, et al. Results of ambulatory electrocardiography in overtly healthy Doberman Pinschers with echocardiographic abnormalities. J Am Vet Med Assoc. 2000;217: 1328-1332.

5. Martin MW, Stafford Johnson MJ, Strehlau G, et al. Canine dilated cardiomyopathy: a retrospective study of prognostic findings in 367 clinical cases. J Small Anim Pract. 2010;51:428-436.

6. Yamada T, Hirashiki A, Cheng XW, et al. Relationship of myocardial fibrosis to left ventricular and mitochondrial function in nonischemic dilated cardiomyopathy - a comparison of focal and interstitial fibrosis. J Card Fail. 2013;19:557-564.

7. Owczarek-Lipska M, Mausberg TB, Stephenson H, et al. A 16-bp deletion in the canine PDK4 gene is not associated with dilated cardiomyopathy in a European cohort of Doberman Pinschers. Anim Genet. 2013;44:239.

8. Meurs KM, Lahmers $S$, Keene BW, et al. A splice site mutation in a gene encoding for PDK4, a mitochondrial protein, is associated with the development of dilated cardiomyopathy in the Doberman pinscher. Hum Genet. 2012;131:1319-1325.

9. Mausberg TB, Wess G, Simak J, et al. A locus on chromosome 5 is associated with dilated cardiomyopathy in Doberman Pinschers. PLoS One. 2011;6:e20042.

10. McCutcheon LJ, Cory CR, Nowack L. Respiratory chain defect of myocardial mitochondria in idiopathic dilated cardiomyopathy of Doberman pinscher dogs. Can J Phys Pharm. 1992;70:1529-1533.

11. Meurs K. 2016 Doberman DCM Update \& DCM2 Gene. NC State Vet Med. 2017. Available online at https://mymediasite.online.ncsu.edu/online/ Play/d84281e7f40643bd84d96f0755f0cb9b1d (accessed December 15, 2017).

12. Jeoung $\mathrm{N}$, Robert $\mathrm{H}$. Role of pyruvate dehydrogenase kinase 4 in regulation of blood glucose levels. Korean Diabetes J. 2010;34: 274-283.

13. Meurs KM, Sunshine $L$, Bruce KW, et al. A splice site mutation in a gene encoding for PDK4, a mitochondrial protein, is associated with the development of dilated cardiomyopathy in the Doberman pinscher. Hum Genet. 2012;131:1319-1325.

14. Lopaschuk GD, Ussher JR, Folmes CD, et al. Myocardial fatty acid metabolism in health and disease. Physiol Rev. 2010;90:207-258.

15. Doenst T, Ngyuen DT, Abel DE, et al. 2013. Cardiac metabolism in heart failure. Circ Res. 2013;113:709-724. 
16. Slee EA, Harte MT, Kluck RM, et al. Ordering the cytochrome c-initiated caspase cascade: hierarchical activation of caspases- $2,-3$, $-6,-7,-8$, and -10 in a caspase-9-dependent manner. J Cell Biol. $1999 ; 144: 281-292$.

17. Sosa I, Estrada AH, Winter BD, et al. In vitro evaluation of mitochondrial dysfunction and treatment with adeno-associated virus vector in fibroblasts from Doberman Pinschers with dilated cardiomyopathy and a pyruvate dehydrogenase kinase 4 mutation. Am J Vet Res. 2016;77: 156-161.

18. Brentnall $M$, Rodriguez-Menocal L, De Guevara R, et al. Caspase-9, caspase- 3 and caspase-7 have distinct roles during intrinsic apoptosis. BMC Cell Biol. 2013;4:32.

19. Swiss R, Niles A, Cali JJ, et al. Validation of a HTS-amenable assay to detect drug-induced mitochondrial toxicity in the absence and presence of cell death. Toxicol In Vitro. 2013;27: 1789-1797.

20. Aslanidi GV, Rivers AE, Ortiz L, et al. Optimization of the capsid of recombinant adeno-associated virus 2 (AAV2) vectors: the final threshold? PLoS One. 2013;8:e59142.

21. Zamaraeva MV, Sabirov RZ, Maeno E, et al. Cells die with increased cytosolic ATP during apoptosis: a bioluminescence study with intracellular luciferase. Cell Death Differ. 2005;12: 1390-1397.
Cite this article as: Taggart $K$, Estrada $A$, Thompson $P$, Lourenco $F$, Kirmani S, Suzuki-Hatano S, Pacak CA (2017) PDK4 deficiency induces intrinsic apoptosis in response to starvation in fibroblasts from Doberman Pinschers with dilated cardiomyopathy, BioResearch Open Access 6:1, 182-191, DOI: 10.1089/biores.2017.0023.

$\begin{aligned} & \text { Abbreviations Used } \\ & \mathrm{AAV}==\text { adeno-associated virus } \\ & \mathrm{CBA}=\text { chick beta actin } \\ & \mathrm{DCM}=\text { dilated cardiomyopathy } \\ & \mathrm{DHE}=\text { dihydroethidium } \\ & \mathrm{DP}=\text { Doberman pinscher } \\ & \mathrm{FBS}=\text { fetal bovine serum } \\ & \mathrm{MOI}=\text { multiplicity of infection } \\ & \mathrm{PDC}=\text { pyruvate dehydrogenase complex } \\ & \mathrm{PDK} 4=\text { pyruvate dehydrogenase kinase } 4 \\ & \mathrm{ROS}=\text { reactive oxygen species }\end{aligned}$

\section{Publish in BioResearch Open Access}

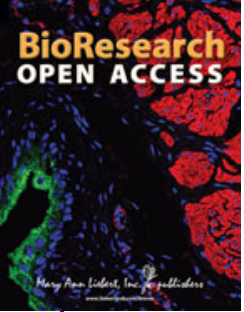

- Broad coverage of biomedical research

- Immediate, unrestricted online access

- Rigorous peer review

- Compliance with open access mandates

- Authors retain copyright

- Highly indexed

- Targeted email marketing

liebertpub.com/biores 